\title{
EFEITOS DE DOSES DE GLYPHOSATE NO CONTROLE DA TIRIRICA (Cyperus rotundus L.)*
}

\author{
N.E. DE M. BELTRÃO**, P.H.F. DE PAULA***, J.F. ALVES***, F.J.F. TAVORA*** \\ * Parte da dissertação apresentada pelo pri - \\ meiro autor ao Departamento de Fitotecnia \\ do Centro de Ciências Agrárias da Univer- \\ sidade Federal do Ceará, para obtenção do \\ grau de Mestre em Agronomia, pela Univ. \\ Fed. do Ceará. \\ ** Pesquisador do CNP-Algodão - EMBRAPA, \\ Campina Grande, PB. \\ *** Professores do Centro de Ciências Agrárias \\ da Universidade Federal do Ceará, Fortale- \\ za, CE.
}

\section{RESUMO}

Em três experimentos instalados em 1975 em Fortaleza e Pentecoste, no Estado do Ceará, procurou-se estudar o ef eito do glyphosate aplicado a $0,1,2,3,4,5$ c $6 \mathrm{~kg}$ do i.a/ha no controle da tiririca (Cyperus rotundus L.), em solo sem cultura.

O produto foi aplicado em pós-emergência quando a tiririca estava próxima do floresci mento e em florescimento. Foi realizada apenas uma aplicação do glyphosate para todos os tratamentos.

Nas condições em que foram realizados es ses experimentos as seguintes conclusões podem ser obtidas: 1 . Doses de 2,00 a $3,00 \mathrm{~kg} / \mathrm{h}$ a do glyphosate ofe receram um satisfatório grau de controle desta planta daninha por 30 dias, com apenas uma aplicação; 2. 0 glyphosate deve ser aplicado quando a tiririca estiver com seis a sete folhas, com alto vigor vegetativo e antes do início do florescimento; 3 . Doses elevadas como $6,00 \mathrm{~kg} / \mathrm{ha}$, não são recomendad as pois, o grau de controle é semelhante aos obtidas com doses menores, como 3,00 kg/ha.

\section{SUMMARY}

EFFECTS OF GLYPHOSATE RATES ON PURPLE NUTSEDGE (Cyperus rotundus L.) CONTROL.

Three experiments were conducted in 1975 at Fortaleza and Pentecoste, Sta te of Ceará, to determine the effectiveness of glyphosate in the control of purple nutsedge (Cyperus rotundus L.) in soil without a growing crop.

The treatments were as follows: Control (without glyphosate); and 6 levels of as 1, 2, 3, 4,5 and $6 \mathrm{~kg}$ of active ingredient per hectare.

The material was applied as a post -emergent when the nutsedge was prior, or in the flowering stage. Only one aplication was made for each of the treatments.

Under the conditionns of these experiments, the following conclusions can be drawn: 1. Glyphosate, at levels of 2 and $3 \mathrm{~kg}$ (ia)/ha, gave a satisfactory degree of control for 30 days with only one application; 2. Glyphosate must be applied when the nutsedge is in the 6-or 7-leaf stage and in rapid vegetative development and before the initiation of flowering; and, 3. Higher levels of application are not recommended because the degree of control is similar to that obtained at $3 \mathrm{~kg}(\mathrm{ia}) / \mathrm{ha}$.

\section{INTRODUÇÃO}

A tiririca (Cyperus rotundus L.) é conderada uma das dez piores plantas daninhas do mundo (19), em virtude de sua alta nocividade (7) e agressividade às culturas, bem como por ser uma espécie de larga amplitude ecológica (10 ).

De acordo com Pinheiro e Krug (17), ela teve sua origem na Nova Zelândia, de onde se espalhou para várias regiões do globo, a ponto de ser hoje, uma espécie cosmopolita, habitando principalmente as regiões tropicais e subtropicais.

Pode ser uma planta possuidora de diversas maneiras de propagação, tanto gâmicamente, por intermédio de suas sementes, como agâmicamente, por meio de órgãos vegetativos subterrâneos, o 
combate a esta ciperácea é extremamente difícil e custoso.

No Brasil, especialmente nas áreas irrigadas do Nordeste referida planta daninha está bastante difundida, atuando a água como um excelente veículo de propagação, além de ser disseminada quando se faz sistematização de terras, sem o cuidado prévio de limpar os implementos agrícolas.

Segundo as observações de Beltrão (4) a fitomassa hipógea do esporófito adulto da espécie em tela, compreende uma formação rizomática que, de distância em distância, se hipertrofia para constituir estruturas semelhantes e tubérculos adquirindo, o conjunto, aspecto de rosário. Já à fitomassa epígea compreende uma haste com aspecto graminoide, porém sem nós, com seção triangular e nas condições mesológicas do Estado do Ceará, apresenta altura de 15 a $50 \mathrm{~cm}$ e ciclo bastante rápido, florescen do em cerca cerca de 15 a 20 dias, após a reatividade dos pontos de crescimento dos tuberculóides.

Em função do seu hábito de crescimento com elevada produção de órgãos subterrâneos, Deutsch (9) a chama de "A pior planta daninha do Mundo".

Vários herbicidas orgânicos e inorgânicos, bem como métodos mecânicos, culturais e biológicos de combate, tem sid o uti lizados no con tro le da tiririca, porém na maioria dos casos sem o suces so esperado.

Horowitz (12), em experimento sobre frequência de cortes em três plantas daninhas perenes, entre as quais a tiririca, verificou que a aludida planta foi mais resistente aos cortes do que o Sorghum halepense (L.) Pers e o Cynodon dactylon L. (Pers ), apresentando elevada taxa de crescimento após diversos cortes.

Outros métodos de controle tem sido testados, como o uso do fogo, porém conforme verificaram Gaskins e Colberg (11), a utilização de lança chamas reduz, porém não erradica a tiririca.

Um dos métodos que vem sendo pes- quis ado é o controle químico por intermédio de herbicidas. Almeida (3) em estudos envolvendo diversos herbicidas em vários locais, verificou que o EPTC em pré-plantio incorporado na dose de $4,0 \mathrm{~kg} / \mathrm{ha}$ exerceu um bom controle sobre a tiririca, e esse tiocarbomato também é recomendado por vários outros autores $(2,18,20)$, autan do destruindo os núcleos celulares e promovendo decomposição do amido, local izado nos "tubé rculos" (6).

Diver sos outros herbicidas tem sido testados no controle à tiririca, tais como 2,4-D, broma cil, vernolate etc., porém o grau de controle desejável, nem sempre é alcan çado.

O presente trabalho teve por objetivo a verificação, em condições de campo, dos efeitos do glyphosate = I N - (fosf onometil )-glicina 1 sobre a tiririca no Estado do Ceará, em áreas irrig idas e não irrig adas.

\section{MATERIAIS E MÉTODOS}

Os experimentos, em número de três, foram instalados no ano agrícola de 1975, no Campo de Experimentação do DNOCS ( solo de textura franca) na $\mathrm{Fa}$ zenda Experimental do Vale do Curú da UFCe ( solo franco arenoso) e no Campus do Centro de Ciências Agrárias da UFCe ( solo de textura areia franca) sendo os dois primeiros localizados no município de Pentecoste e o último em Fortaleza, todos no Estado do Ceará, Brasil.

Vinte dias antes da aplicação do herbicida, procedeu-se aração e gradagem nos materiais edáficos naturalmente infestados pela tiririca, sendo que para o ensaio no Campo de Experimentacão do DNOCS, em seguida a área foi sulcada e irrigada pelo método da infiltração.

Para os três experimentos, no momento da aplic ação do glyph osate havia pouco vento, menos do que $6 \mathrm{~km} / \mathrm{hora}$, ausência de precipitação pluvial, céu limpo e a temperatura do ar estava em torno de $30^{\circ} \mathrm{C}$.

A população da planta daninha con - 
siderada, apresentava-se com altura média de 6 a $8 \mathrm{~cm}$, em período de pré-floração e exibindo exuberante vigor vegetativo, nos locais 1 ((DNOCS) e 2 ("Campus "da UFCe ). Com relação ao ensaio do local 3 ( Fazenda Experimental do Vale do Curú) o herbicida foi aplicado quando havia cerca de $15 \%$ de plantas já em estádio de floração e a altura média das plantas era de $7 \mathrm{~cm}$.

Em todos os experimentos foram testados os seguintes tratamentos : testemunha (sem herbicida); e glyphosate a 1,0, 2,0, 3,0, 4,0, 5,0 e 6,0 kg do i.a/ha.

$\mathrm{O}$ delin eamen to estatístico utili zado foi o de blocos ao acaso com quatro repetições, sendo de $12 \mathrm{~m}^{2}(2 \mathrm{~m} \times 6 \mathrm{~m})$ a área de cada unidade experimental e de $6 \mathrm{~m}^{2}$ $(1 \mathrm{~m} \times 6 \mathrm{~m})$ a área útil.

O produto testado glyphosate IN (fosfonometil) glicinal 1, sendo Jaworski (13) é translocável, especialmente por via lep tomática e provavelmente atu a inibindo o camin ho bioss intético do ácido amino aromático, rota para a síntese da fenilalanina, aminoácido protéico.

Para a aplicação do produto, utilizouse um pulverizador portátil de $\mathrm{CO}_{2}$, pressão constante de $30 \mathrm{lb} / \mathrm{pol}^{2}$, vazão de 400 1/ha e munido com quatro bicos tipo teejet 80.04. Realizou-se somente uma aplicação por unidade experimental.

A avaliação do efeito-herbicida foi efetuada 15 e 30 dias após a aplicação do glyphosate, sendo procedida de maneira qualitativa, através de avaliação visual, segundo método de Alves (1) e quantitativa, envolvendo dois métodos sinecológicos (contagem de plantas por unidade de área e peso da fitomassa epigea fresca ). Tais procedimentos foram realizados numa área correspondente a 8,37\% da área útil da parcela.

Os resultados obtidos foram analisados estatisticamente pelos métodos convencionais segundo a técnica descrita por Cochran e Cox (8) e os dados referentes as determinações de contagem do número de plantas foram transformados para $\mathrm{V} x$ +1 de acordo com Brilho (5).

\section{RESULTADOS E DISCUSSÃO}

AVALIACẼO 15 DIAS APÓS A
APLICACÃO DO GLYPHOSATE

Considerando a avaliação visual nos quadros 1 e 2 observa-se para os três experiment os que o grau de controle da tiririca já foi bastante satisfatório a partir de dosagens de $2,0 \mathrm{~kg}$ i.a/ha, fato também verificado na densidade de plantas, com alguma diferenças no nível de controle devido as variações microclimáticas de cada local onde o trabalho foi executado, o que está de aco rdo com as con siderações de Muzik \& Mauldin (16) sobre a influência dos fatores ambientais na resposta das plantas aos herbicidas.

No ensaio conduzido no Campo de Experimentação do DNOCS único local onde determinou-se o peso fresco de plantas ( fitomassa epígea ), verifica-se no quadro 1 que as dosagens de 2 a $6 \mathrm{~kg}$ i.a/ha não diferiram estatisticamente pelo teste Tukey ao nível de $5 \%$ de probabilidade, fato que não ocorreu com relação a conta ge m de plantas numa superfície de $0,5 \mathrm{~m}^{2}$ ( $8,34 \%$ da área útil ), onde a dose de $2,0 \mathrm{~kg}$ i.a/ha foi estatisticamente inferior as mais elevadas (5 e $6 \mathrm{~kg}$ i.a /ha ). Tal fato provavelmente ocorreu devido que para efeito de contagem de plantas, considerou-se apenas aquelas que se apresentavam aparentemente norma is com folhas $e$ caules verdes, fotossintetizando. Porém, apesar do mesmo critério ter sido utilizado para peso fresco, algumas plantas que visualmente apresentavam-se normais, na realidade estavam com o metabolis mo comprometido devido ao efeito do glyphosate, o que resultou na variação do retro referido parâmetro computado.

Observando-se conjuntamente as três variáveis estudadas nos três locais, verificase que uma dose de 2 a $3 \mathrm{~kg}$ i.a/ha já é suficiente para um elevado grau de controle, devendo-se considerar que os ensaios foram conduzidos sem cultura, logo sem o chamado controle cultural, especialmente por luz, uma vez que a tiririca é uma planta de meabolismo C4 (19) e altamente exigenta em luz (14). Assim, 
Quadro 1 - Efeito do glyphosate no controle da tiririca ( $C$. rotundus, L.) 15 dias após a aplicação. Experimento 1, Campo de Experi mentação do DNOCS - Pentecoste, Ceará, Brasil, 1975.

\begin{tabular}{|c|c|c|c|c|c|}
\hline \multirow[b]{2}{*}{ TRATAMENTOS } & \multirow{2}{*}{$\begin{array}{c}\text { Avaliação } \\
\text { Visual }\end{array}$} & \multicolumn{2}{|c|}{ CONTAGEM DE PLANTAS } & \multicolumn{2}{|c|}{ PESO FRESCO DE PLANTAS } \\
\hline & & $\begin{array}{l}\text { Médias dos tratamentos } \\
\text { (Trans. } V^{\bar{x}+1)}\end{array}$ & $\begin{array}{l}\% \text { de Controle } \\
\text { (Dados originais) }\end{array}$ & $\begin{array}{c}\text { Médias dos tratamentos } \\
(\mathrm{g})\end{array}$ & $\begin{array}{l}\% \text { de Controle } \\
\text { (Dados originais) }\end{array}$ \\
\hline $\begin{array}{l}\text { TESTEMUNHA } \\
1 \mathrm{~kg} \text { i.a/ha } \\
2 \mathrm{~kg} \text { i.a/ha } \\
3 \mathrm{~kg} \text { i.a/ha } \\
4 \mathrm{~kg} \text { i.a/ha } \\
5 \mathrm{~kg} \text { i.a/ha } \\
6 \mathrm{~kg} \text { i.a/ha }\end{array}$ & $\begin{array}{r}0,00 \mathrm{a} \\
8,07 \mathrm{~b} \\
9,50 \mathrm{c} \\
9,80 \mathrm{c} \\
9,90 \mathrm{c} \\
10,00 \mathrm{c} \\
10,00 \mathrm{c}\end{array}$ & $\begin{array}{r}10,10 \mathrm{a} \\
5,52 \mathrm{~b} \\
3,80 \mathrm{c} \\
2,98 \mathrm{~cd} \\
2,63 \mathrm{~cd} \\
1,87 \mathrm{~d} \\
1,49 \mathrm{~d}\end{array}$ & $\begin{array}{r}0,00 \\
70,42 \\
86,55 \\
91,44 \\
94,13 \\
97,31 \\
98,77\end{array}$ & $\begin{array}{l}30,25 \mathrm{a} \\
8,67 \mathrm{~b} \\
3,70 \mathrm{bc} \\
2,67 \mathrm{bc} \\
1,75 \mathrm{c} \\
0,77 \mathrm{c} \\
0,25 \mathrm{c}\end{array}$ & $\begin{array}{r}0,00 \\
71,32 \\
87,77 \\
91,16 \\
94,21 \\
97,44 \\
99,17\end{array}$ \\
\hline
\end{tabular}

OBS.: Duas médias não seguidas pela mesma letra diferem estatisticamente ao nível de $5 \%$ de probabilidade pelo teste de Tukey.

Quadro 2 - Efeito do glyphosate no controle da tiririca (C. rotundus, L.) 15 dias após a aplicação. Experimentos 2 e 3 , respectivamente localizados no "Campus" do Centro de Ciências Agrárias (CCA) da UFC, Fortaleza, Ceará, Brasil, e Fazenda Experimental do Vale do Curú, Pentecoste, Ceará, Brasil, 1975.

\begin{tabular}{|c|c|c|c|c|c|c|}
\hline \multirow{3}{*}{ TRATAMENTOS } & \multirow{3}{*}{$\begin{array}{c}\text { Avaliação } \\
\text { Visual }\end{array}$} & \multicolumn{3}{|c|}{ EXPERIMENTO 2} & \multicolumn{2}{|c|}{ EXPERIMENTO 3} \\
\hline & & \multicolumn{2}{|c|}{ CONTAGEM DE PLANTAS } & \multirow[b]{2}{*}{$\begin{array}{c}\text { Avaliação } \\
\text { Visual }\end{array}$} & \multicolumn{2}{|c|}{ CONTAGEM DE PLANTAS } \\
\hline & & $\begin{array}{l}\text { Médias dos tratamentos } \\
\quad \text { (transf. } V^{\left.\frac{x}{x+1}\right)}\end{array}$ & $\begin{array}{l}\% \text { de Controle } \\
\text { ((Dados } \\
\text { originais) }\end{array}$ & & $\begin{array}{l}\text { Médias dos tratamentos } \\
\left.\quad \text { (transf. } V^{\frac{x}{x}+1}\right)\end{array}$ & $\begin{array}{l}\text { \% de Controle } \\
\text { ((Dados } \\
\text { originais) }\end{array}$ \\
\hline $\begin{array}{l}\text { TESTEMUNHA } \\
1 \mathrm{~kg} \text { i.a/ha } \\
2 \mathrm{~kg} \text { i.a/ha } \\
3 \mathrm{~kg} \text { i.a/ha } \\
4 \mathrm{~kg} \text { i.a/ha } \\
5 \mathrm{~kg} \text { i.a/ha } \\
6 \mathrm{~kg} \text { i.a/ha }\end{array}$ & $\begin{array}{r}0,00 \mathrm{a} \\
5,87 \mathrm{~b} \\
9,00 \mathrm{c} \\
9,37 \mathrm{c} \\
9,75 \mathrm{c} \\
9,87 \mathrm{c} \\
10,00 \mathrm{c}\end{array}$ & $\begin{array}{l}8,40 \mathrm{a} \\
4,65 \mathrm{~b} \\
2,27 \mathrm{c} \\
1,61 \mathrm{c} \\
1,39 \mathrm{c} \\
1,30 \mathrm{c} \\
1,28 \mathrm{c}\end{array}$ & $\begin{array}{r}0,00 \\
65,94 \\
93,55 \\
97,49 \\
98,56 \\
98,92 \\
98,92\end{array}$ & $\begin{array}{l}0,00 \mathrm{a} \\
7,05 \mathrm{~b} \\
7,75 \mathrm{c} \\
8,65 \mathrm{~d} \\
9,27 \mathrm{e} \\
9,75 \mathrm{e} \\
9,87 \mathrm{e}\end{array}$ & $\begin{array}{r}13,92 \mathrm{a} \\
9,20 \mathrm{~b} \\
6,19 \mathrm{c} \\
4,44 \mathrm{~d} \\
3,67 \mathrm{de} \\
3,02 \mathrm{de} \\
2,64 \mathrm{e}\end{array}$ & $\begin{array}{r}0,00 \\
56,01 \\
80,59 \\
89,78 \\
93,27 \\
95,73 \\
96,76\end{array}$ \\
\hline
\end{tabular}

OBS.: Duas médias não seguidas pela mesma letra diferem estatisticamente ao nível de $5 \%$ de probabilidade pelo teste de Tukey. 
Quadro 3 - Efeito do glyphosate no controle da tiririca ( $C$. rotundus, L.) 30 dias após a aplicaçāo. Experimento 1, Campo de Experimentação do DNOCS - Pentecoste, Ceará, Brasil, 1975.

\begin{tabular}{|c|c|c|c|c|c|}
\hline \multirow[b]{2}{*}{ TRATAMENTOS } & \multirow{2}{*}{$\begin{array}{l}\text { Avaliação } \\
\text { Visual }\end{array}$} & \multicolumn{2}{|c|}{ CONTAGEM DE PLANTAS } & \multicolumn{2}{|c|}{ PESO FRESCO DE PLANTAS } \\
\hline & & $\begin{array}{l}\text { Médias dos tratamentos } \\
\quad\left(\text { transf. } \vee \frac{1}{x+1}\right)\end{array}$ & $\begin{array}{l}\% \text { de Controle } \\
\text { (Dados originais) }\end{array}$ & $\begin{array}{c}\text { Médias dos tratamentos } \\
(\mathrm{g})\end{array}$ & $\%$ de Controle \\
\hline $\begin{array}{l}\text { TESTEMUNHA } \\
1 \mathrm{~kg} \text { i.a/ha } \\
2 \mathrm{~kg} \text { i.a/ha } \\
3 \mathrm{~kg} \text { i.a/ha } \\
4 \mathrm{~kg} \text { i.a/ha } \\
5 \mathrm{~kg} \text { i.a/ha } \\
6 \mathrm{~kg} \text { i.a/ha }\end{array}$ & $\begin{array}{l}0,00 \mathrm{a} \\
7,15 \mathrm{~b} \\
8,30 \mathrm{c} \\
8,87 \mathrm{~cd} \\
9,17 \mathrm{~d} \\
9,00 \mathrm{~cd} \\
9,30 \mathrm{~d}\end{array}$ & $\begin{array}{l}7,12 \text { a } \\
5,37 \text { a } \\
3,48 \text { b } \\
3,11 \text { b } \\
3,10 \mathrm{~b} \\
1,99 \mathrm{~b} \\
1,82 \\
1, \mathrm{~b}\end{array}$ & $\begin{array}{r}0,00 \\
45,09 \\
77,94 \\
81,86 \\
82,35 \\
94,12 \\
95,09\end{array}$ & $\begin{array}{r}14,87 \mathrm{a} \\
8,42 \mathrm{~b} \\
3,35 \mathrm{c} \\
2,70 \mathrm{c} \\
2,57 \mathrm{c} \\
0,87 \mathrm{c} \\
0,67 \mathrm{c}\end{array}$ & $\begin{array}{r}0,00 \\
43,36 \\
77,48 \\
81,85 \\
82,69 \\
94,11 \\
95,46\end{array}$ \\
\hline
\end{tabular}

OBS.: Duas médias não seguidas pela mesma letra diferem estatisticamente ao nível de $5 \%$ de probabilidade pelo teste de Tukey.

Quadro 4 - Efeito do glyphosate no controle da tiririca ( $C$. rotundus, L.) 30 dias após a aplicaçäo. Experimentos 2 e 3, respectivamente localizados no "Campus" do Centro de Ciências Agrárias (CCA) da UFC, Fortaleza, Ceará, Brasil, e Fazenda Experimental do Vale do Curú, Pentecoste, Ceará, Brasil, 1975.

\begin{tabular}{|c|c|c|c|c|c|c|}
\hline \multirow[b]{3}{*}{ TRATAMENTOS } & \multirow{3}{*}{$\begin{array}{c}\text { Avaliação } \\
\text { Visual }\end{array}$} & \multicolumn{3}{|c|}{ EXPERIMENTO 2} & \multicolumn{2}{|c|}{ EXPERIMENTO 3} \\
\hline & & \multicolumn{2}{|c|}{ CONTAGEM DE PLANTAS } & \multirow[b]{2}{*}{$\begin{array}{c}\text { Avaliaçāo } \\
\text { Visual }\end{array}$} & \multicolumn{2}{|c|}{ CONTAGEM DE PLANTAS } \\
\hline & & $\begin{array}{l}\text { Médias dos tratamentos } \\
\left.\quad \text { (transf. } \vee^{\bar{x}+1}\right)\end{array}$ & $\begin{array}{l}\% \text { de Controle } \\
\text { ((Dados } \\
\text { originais) }\end{array}$ & & $\begin{array}{l}\text { Médias dos tratamentos } \\
\text { (transf. } \sqrt{x+1} \text { ) }\end{array}$ & $\begin{array}{l}\% \text { de Controle } \\
\text { ((Dados } \\
\text { originais) }\end{array}$ \\
\hline $\begin{array}{l}\text { TESTEMUNHA } \\
1 \mathrm{~kg} \mathrm{i.a} / \mathrm{ha} \\
2 \mathrm{~kg} \text { i.a/ha } \\
3 \mathrm{~kg} \mathrm{i.a} / \mathrm{ha} \\
4 \mathrm{~kg} \text { i.a } / \mathrm{ha} \\
5 \mathrm{~kg} \text { i.a/ha } \\
6 \mathrm{~kg} \mathrm{i.a} / \mathrm{ha}\end{array}$ & $\begin{array}{l}0,00 \mathrm{a} \\
7,47 \mathrm{~b} \\
8,62 \mathrm{~cd} \\
8,47 \mathrm{c} \\
9,30 \mathrm{~d} \\
9,52 \mathrm{~d} \\
9,87 \mathrm{~d}\end{array}$ & $\begin{array}{l}8,41 \mathrm{a} \\
4,96 \mathrm{~b} \\
3,63 \mathrm{bc} \\
3,43 \mathrm{bc} \\
2,40 \mathrm{c} \\
3,39 \mathrm{bc} \\
2,25 \mathrm{c}\end{array}$ & $\begin{array}{r}0,00 \\
66,07 \\
81,78 \\
81,78 \\
92,14 \\
82,86 \\
91,43\end{array}$ & $\begin{array}{l}0,00 \mathrm{a} \\
5,22 \mathrm{~d} \\
5,32 \mathrm{bc} \\
6,02 \mathrm{~cd} \\
6,50 \mathrm{de} \\
7,00 \mathrm{e} \\
6,75 \mathrm{de}\end{array}$ & $\begin{array}{r}14,13 \mathrm{a} \\
7,82 \mathrm{~b} \\
6,16 \mathrm{c} \\
5,91 \mathrm{c} \\
5,65 \mathrm{c} \\
5,51 \mathrm{c} \\
5,24 \mathrm{c}\end{array}$ & $\begin{array}{r}0,00 \\
69,34 \\
81,40 \\
82,78 \\
84,42 \\
85,05 \\
86,43\end{array}$ \\
\hline
\end{tabular}

OBS.: Duas médias não seguidas pela mesma letra diferem estatisticamente ao nivel de $5 \%$ de probabilidade pelo teste de Tukey. 
no caso de aplicação com a cultura, desde que se obtenha seletividade agronômica, o controle poderá ser ainda maior.

\section{APLICACÃO DO GLYPHOSATE} AVALIA̧ÇÃO 30 DIAS APÓS A

Nesta segunda avaliação as magnitudes de controle, embora um pouco inferiores à avaliação de 15 dias, mantiveram-se satisfatórias, conforme mostram os quadros e 3 e 4 . No entanto, verificouse que no ensaio conduzido na Fazenda Experimental do Curti, os percentuais de controle, especialmente nas doses mais elevadas foram inferiores aos demais locais testados. Tal fato, está relacionado com o padrão de translocação de fotossintato, uma vez que naquele local, quando da aplicação do herbicida cerca de $15 \%$ da população de tiririca encontravase em estádio de florescimento e como o glyphosate aplicado às folhas é translocado via floema para a fitomassa hipógea do esporórito e estando o movimento de metabólitos mais acrópeto do que basípeto, o herbicida não chegou na mesma quantidade nos órgãos subterrâneos da planta, como possivelmente ocorreu nas demais situações, onde estando a população em pleno período vegetativo, a corrente floemática estava em maior intensidade basipetalmente, levando metabólitos para o crescimento e armazenamento nos órgãos subterrâneos da espécie em estudo, trazendo consigo o herbicida. Tais resultados confirmam as recomendações de Muzik (15) sobre a direção do movimento de fotossintato pelo tecido vascular da planta e o controle, especialmente de plantas polacantas.

\section{LITERATURA CITADA}

1. Alves, A. O efeito-herbicida em ervas daninhas. Atualidades Agronômicas, Maio/Junho. 1974. p. 34-36.

2. Almeida, F. de S. \& Fonseca, A.M. Efeito do herbicida EPTC no "Controle do Cyperus rotundus L.". Agron. Mocamb. 1 (4): 215. 221, 1967.

3. Almeida F. de S. Controle da "tiririca", Cyperus rotundus e $C$. esculentus. (con- tribuição para o seu estudo). Agron. Mocamb. 6 (2): 149-155, 1972.

4. Beltrāo, N.E. de M. A tiririca (Cyperus rotundus, L.). Caracteristicas e controle. Centro de Ciências Agrárias. U.F.Ce. 1975. $10 \mathrm{p}$.

5. Brilho, C.C. Análise de contagens de ervas em ensaios com herbicidas. In: Anais do IV Seminário Brasileiro de Herbicidas $e$ Ervas Daninhas. 1962. p. 449-455.

6. Botha, P.J. \& Nel, P.C. The effect of EPTC an the anatomy of brow nutsedge (Cype. rus rotundus, L.). Agroplanta, 4 (3): 57-62, 1972.

7. Cardenas, J., Reyes, P.C. \& Doll, J.D. Tropical weeds; Malezas Tropicales. Bogotá, Colombia. Instituto Colombiano Agrope. cuário. Vol. 1. 1972. p. 75-76.

8. Cochran, W.G. \& Cox, G.M. Experimental Designs. Second Edition. John Wiley \& Sons, Inc. London. 1975. p. 106-114.

9. Deutsch, A. Cyperus rotundus. The world's worst weed and what's being done about it. World Forming. 1973. p. 12-13.

10. Fisher, H. Origem, distribuição, thabitat e problemas especiais de ervas daninhas. In: Warren, G.F. et al, "Curso intensivo de controte de ervas daninhas". Viçosa, Universidade Federal de Viçosa. 1973. p. 11-15.

11. Gaskins, M.H. \& Colberg, C.M. Controlling pudple nutsedge in the wet tropics by burning and sading. Proc. Fla. Sta. Hort. Soc., 83: 367-369, 1970.

12. Horowitz, M. Effects of frequent dipping os the perenial weeds, Cynodon dactylon (L.) Pers., Sorghum halepense (L.) and Cype. rus rotundus, L. Exp. Agric., 8 (3): 225-234, 1972.

13. Jaworski, E.G. Mode of action of $\mathbf{N}-$ phosphonomethyl glycine Inhibition of aromatic amino acid biosynthesis. J. Agr. Food chem. 20 (6): 1195-1198, 1972.

14. Magalhães, A.C. Observaçóes sobre o efeito da luz no crescimento da tiririca, Cyperus rotundus, L. Bragantia. 26 (9): 131-142, 1967. 
15. Muzik, T.J. Weed biology and control. Mc Graw-Hill Book Campany. 1970. p. 59,60.

16. Muzik, T.J. \& Mauldin, W.G. Influence of environment on the respanse of plants to herbicides. Weeds, 12: 142-145, 1964.

17. Pinheiro, J.V. \& Krug, H.P. Alguns herbicidas modernos no controle da tiririca (Cyperus rotundus, L.). Boletim 3, 2.: Edição. Companhia Paulista de Estradas de Ferro Rio Claro, SP. 1956, 16 p.

18. Sacco, J.C.; Forseer, R.; Deuber, R.; Leiderman, L.; Grassi, N.; Costa, A.M.; Xavier,
F.E.; Andrade, V.A.; Coelho, J.P. \& Silva, J.B. Controle químico das plantas invasoras da cultura do feijăo. Comissão $\mathrm{Na}$ cional de Herbicidas e Ervas Daninhas. 1973, 7 p. Indicação da Pesquisa 5.

19. Wiliam, R.D. Fisiologia das plantas eficientes (C4) e ineficientes (C3). In: Warren, G.F. et al. Curso intensivo de controle de ervas daninhas. Viçosa, Universidade Federal de Viçosa. 1973. p. 168-179.

20. Zanardi, D.; Piras, S. \& Deligia, S. Cyperus rotundus. Infestante dell' agmmento possibilita di lotta. Not mal Piante. 85: 161-180, 1971. 